\title{
Expression of PBMC apoptosis-related factors in patients with chronic hepatitis $B$ and their relationships with clinical prognosis
}

\author{
LI GUO and YANLI LIANG \\ Department of Liver Disease, Xuzhou Infectious Disease Hospital, Xuzhou, Jiangsu 221000, P.R. China
}

Received May 8, 2017; Accepted September 18, 2017

DOI: $10.3892 /$ etm.2017.5299

\begin{abstract}
The present study was conducted to investigate the expression of apoptosis-related factors in peripheral blood mononuclear cells (PBMC) of patients with chronic hepatitis B and their correlation to clinical prognosis. Sixty-two patients that were diagnosed with chronic hepatitis B were admitted to Xuzhou Hospital from March 2015 to February 2016 and were enrolled as the observation group, while 60 healthy subjects who were examined in the health examination center were selected as the control group. The PBMC of patients were collected, and mRNA expression levels of the apoptotic molecules (FAS, CASP3, CASP8 and CASP9) were measured using real-time fluorescence quantitative PCR. The protein expression levels of apoptosis-related genes in the plasma were detected using enzyme-linked immunosorbent assay (ELISA) and the serum HBV-DNA was quantitatively measured using real-time fluorescence quantitative PCR. The mRNA and protein expression levels of FAS, CASP3, CASP8 and CASP9 in the observation group were significantly higher than those in the control group $(\mathrm{P}<0.05)$. The positive rate and log value of the copy amount of HBV DNA in the observation group were significantly higher than those in the control group $(\mathrm{P}<0.05)$. The Pearson correlation coefficient analysis showed that FAS, CASP3, CASP8 and CASP9 were positively correlated with HBV DNA $(\mathrm{P}<0.05)$. The mRNA expression levels of FAS, CASP3, CASP8 and CASP9 in patients with negative HBV-DNA were significantly lower than those with positive HBV-DNA $(\mathrm{P}<0.05)$. Apoptosis of PBMCs play an important role in the occurrence and development of chronic hepatitis B, and is closely correlated to the level of serum virus replication and prognosis.
\end{abstract}

\section{Introduction}

Hepatitis B (HBV) is a viral infectious disease that is mainly transmitted through contact with blood and body fluids of

Correspondence to: Dr Yanli Liang, Department of Liver Disease, Xuzhou Infectious Disease Hospital, 300 Shuangyong Road, Xuzhou, Jiangsu 221000, P.R. China

E-mail: liangyanli78@163.com

Key words: peripheral blood mononuclear cells, apoptosis-related gene, chronic hepatitis B, prognosis infected people. Currently, there are more than 350 million people worldwide that are chronically infected with $\mathrm{HBV}$, which is the leading cause of cirrhosis and liver cancer. As many as 600,000 people die from hepatitis B each year, which is one of the most serious health problems in the world (1). Although the prevalence of chronic hepatitis B (CHB) has been controlled to below $0.5 \%$ in low endemic areas, it is still more than $10 \%$ in some Asian and western pacific countries (2). Mother-to-child infection is the most common route of transmission areas of high $\mathrm{CHB}$ prevalence. Young people are the main infected population in low endemic areas. Although HBV infection has been reduced through the HBV vaccination program, there are still a large number of people that are infected with HBV or carry the HBV virus (3). Gene type, virus load and immune response function are important factors in HBV infection and chronicity. Therefore, the reduction of immune response function is the main reason of continuous replication of HBV in the body and the increasing viral load (4). Peripheral blood mononuclear cells (PBMC) play an important role in the process of antiviral immune response, and excessive apoptosis of PBMC after viral infection leads to the reduction of the body's antiviral immune response function and an increase of virus load $(5,6)$. However, the apoptotic mechanism of PBMC remains unclear. The present study aims to investigate the expression of apoptosis-related factors in PBMC of patients with $\mathrm{CHB}$ and their relationships with clinical prognosis in order to demonstrate its significance in the chronicity of HBV infection.

\section{Materials and methods}

Sample selection. Sixty-two patients with CHB that were admitted to Xuzhou Hospital between March 2015 and February 2016 were enrolled as the observation group. The inclusion criteria were as follows: i) patients who conform to the diagnostic criteria of chronic hepatitis B; ii) patients who had not been treated with antiviral therapy before admission; and iii) patients who signed the informed consent. The exclusion criteria were as follows: i) patients with hepatitis A, C, D or E; ii) patients with malignant tumor; and iii) women during gestation or lactation. In addition, 60 healthy subjects who were examined in the health examination center at our hospital and had no genetic relationship with patients in the observation group were selected as the control group. The differences 
Table I. General data of research subjects.

\begin{tabular}{|c|c|c|c|c|}
\hline Items & Observation group $(n=62)$ & Control group $(n=60)$ & $t / \chi^{2}$ & P-value \\
\hline Sex (male/female) & $39 / 23$ & $37 / 23$ & 0.020 & 0.888 \\
\hline Age (years) & $20-59$ & $20-60$ & & \\
\hline Average age (years) & $48.85 \pm 7.89$ & $48.37 \pm 7.48$ & 0.345 & 0.731 \\
\hline \multicolumn{5}{|l|}{ Educational level } \\
\hline Junior high school and below & $15(24.19)$ & $13(21.67)$ & 0.505 & 0.777 \\
\hline High school and technical secondary school & $29(46.77)$ & $26(43.33)$ & & \\
\hline Junior college or above & $18(29.03)$ & $21(35.00)$ & & \\
\hline
\end{tabular}

Table II. FAS, CASP3, CASP8 and CASP9 primer sequences.

\begin{tabular}{ll}
$\begin{array}{l}\text { Microsatellite } \\
\text { site }\end{array}$ & \multicolumn{1}{c}{ Sequence } \\
\hline FAS & F: 5'-TCTGGTTCTTACGTCTGTTGC-3' \\
& R: 5'-CTGTGCAGTCCCTAGCTTTCC-3' \\
CASP3 & F: 5'-CAGTGGAGGCCGACTTCTTG-3' \\
& R: 5'-TGGCACAAAGCGACTGGAT-3' \\
CASP8 & F: 5'-GCAAACTGGATGATGACATGAA-3' \\
& R: 5'-TCTTTTCAGGATGTCCAACTTTC-3' \\
CASP9 & F: 5'-GGACATCCAGCGGGCAGG-3' \\
& R: 5'-TCTAAGCAGGAGATGAACAAAGG-3' \\
\hline
\end{tabular}

F, forward; R, reverse.

in characteristics between subjects in the two groups were comparable and not statistically significant $(\mathrm{P}<0.05)$, as shown in Table I. The study was approved by the Ethics Committee of Xuzhou Infectious Disease Hospital and informed consents were signed by the patients and/or guardians.

Sample collection. A total of 3-5 $\mathrm{ml}$ of fasting peripheral venous blood was collected from the research subjects and placed into an ethylenediamine tetracetic acid (EDTA) anticoagulant tube. One of the tubes was separated and stored at $-20^{\circ} \mathrm{C}$, and another was added to $4 \mathrm{ml}$ of lymphocyte separation solution and centrifuged at 2,961 x g for $20 \mathrm{~min}$. The PBMC was taken and stored at $-80^{\circ} \mathrm{C}$.

Detection of mRNA expression levels of apoptotic molecules in PBMC. The extraction of total RNA is as follows. After PBMC was thawed, total RNA was extracted according to the strict operation of the TRIzol reagent instructions. The concentration and purity of RNA were detected, and the concentration was ensured to be within 1.8-2.2. For the primer design, the experimental primers were designed and synthesized by the Shenzhen BGI (Shenzhen, China) and the related primer sequences are shown in Table II. The total tissue RNA was amplified by the DNA target fragment through the access RT-PCR system. The amplification conditions were denaturation at $42^{\circ} \mathrm{C}$ for $5 \mathrm{~min}, 95^{\circ} \mathrm{C}$ for $10 \mathrm{sec}, 95^{\circ} \mathrm{C}$ for $5 \mathrm{sec}$ and $60^{\circ} \mathrm{C}$ for $20 \mathrm{sec}$, a total of 40 cycles, $4^{\circ} \mathrm{C}$ for $2 \mathrm{~h}$ to the end. The electrophoresis was conducted for PCR production, and the electrophoresis result was analyzed by GeneMapper 3.0 software.

Through ethidium bromide (ETBR) staining and 2\% agarose gel electrophoresis, the PCR product was observed by a UV-2000 Ultraviolet Analyzer (Shanghai Scientific Instrument Factory, Shanghai, China). The quantitative analysis was performed by an HMIAS-2000 high-definition color medical graphic analysis system, and the gray ratios of FAS, CASP3, CASP8 and CASP9 mRNA to glyceraldehyde-3-phosphate dehydrogenase (GAPDH) were adopted to represent the relative expression levels of FAS, CASP3, CASP8 and CASP9 mRNA.

Detection of protein expression levels of apoptotic molecules in PBMC. The protein expression levels of FAS, CASP3, CASP8 and CASP9 in the plasma of samples were respectively detected by ELISA, and the related kits were provided by the Nanjing Jiancheng Bioengineering Institute (Nanjing, China). The experimental steps were as follows. First, for dilution and application of the sample, the sample was thawed, and added to the washing buffer for dilution, which was followed by placing the micropore into the reaction plate. Next, horseradish peroxidase was added to the sample and incubated at $37^{\circ} \mathrm{C}$ for $60 \mathrm{~min}$. After the sample was incubated, the plate was washed four times, and placed static for $15 \mathrm{sec}$ during the interval. The chromogenic agent $\mathrm{A}$ and B solution (50 $\mu \mathrm{l}$ for each) was added, respectively, and mixed uniformly. Then, it was incubated at $20^{\circ} \mathrm{C}$ in the dark for $15 \mathrm{~min}$, which was followed by adding $50 \mu \mathrm{l}$ of stop buffer. The OD value at the wavelength $450 \mathrm{~nm}$ was read by a microplate reader (Jiangsu Potebio Co., Ltd., Jiangsu, China) within $15 \mathrm{~min}$, and concentration of FAS, CASP3, CASP8 and CASP9 was calculated.

The 62 patients with CHB were administered with interferon antiviral therapy according to their specific conditions, depending on whether there was a response and tolerance to drugs. The patients were grouped according to the positive conditions of HBV DNA after treatment, and then 3-5 $\mathrm{ml}$ of peripheral venous blood was collected again for the detection of protein expression levels of FAS, CASP3, CASP8 and CASP9.

Detection of HBV DNA. The HBV DNA was detected by using real-time immunofluorescence quantitative PCR method. The related kits were provided by the Sansure Biotech Co., Ltd. Changsha, China), and determined by the professional staff of 
Table III. Comparisons of mRNA expression levels of apoptotic molecules in PBMC between the two groups.

\begin{tabular}{lccccc}
\hline Group & Cases & FAS mRNA & CASP3 mRNA & CASP8 mRNA & CASP9 mRNA \\
\hline Observation group & 62 & $2.59 \pm 0.27$ & $1.95 \pm 0.26$ & $2.12 \pm 0.25$ & $2.23 \pm 0.27$ \\
Control group & 60 & $1.04 \pm 0.18$ & $0.98 \pm 0.17$ & $1.03 \pm 0.14$ & $1.05 \pm 0.14$ \\
t-value & & 24.303 & 29.579 & 30.154 \\
P-value & $<0.05$ & $<0.05$ & $<0.05$ & $<0.05$ \\
\hline
\end{tabular}

Table IV. Comparisons of protein expression levels of apoptotic molecules in PBMC between the two groups (ng/ml).

\begin{tabular}{lccccc}
\hline Group & Cases & FAS & CASP3 & CASP8 & CASP9 \\
\hline Observation group & 62 & $4.59 \pm 0.73$ & $7.54 \pm 0.82$ & $2.83 \pm 0.51$ & $3.32 \pm 0.56$ \\
Control group & 60 & $2.14 \pm 0.32$ & $3.78 \pm 0.74$ & $1.28 \pm 0.42$ & $1.86 \pm 0.43$ \\
t-value & & 23.870 & 26.561 & 18.292 & 16.114 \\
P-value & & $<0.05$ & $<0.05$ & $<0.05$ & $<0.05$ \\
\hline
\end{tabular}

Table V. Comparisons of positive rate of serum HBV DNA and log value of copy amount of HBV DNA between the two groups.

\begin{tabular}{lccc}
\hline Group & Cases & $\begin{array}{c}\text { Positive rate } \\
\text { of HBV DNA }\end{array}$ & $\begin{array}{c}\text { Log value of } \\
\text { copy amount } \\
\text { of HBV DNA }\end{array}$ \\
\hline Observation group & 62 & $56(90.32)$ & $5.83 \pm 0.56$ \\
Control group & 60 & $1(1.67)$ & $1.16 \pm 0.43$ \\
t-value & & 92.373 & 43.816 \\
P-value & & $<0.05$ & $<0.05$ \\
\hline
\end{tabular}

the test center in Xuzhou Hospital. The positive HBV DNA was judged as $\geq 5.0 \times 10^{2}$ copies $/ \mathrm{ml}$.

Statistical analysis. SPSS 19.0 (SPSS, Inc., Chicago, IL, USA) software was used to process data. The logarithmic conversion was adopted for HBV DNA copy number. The measurement data were expressed as mean \pm standard deviation (SD), and enumeration data were expressed by percentage. The chisquare test was utilized and the correlation was assessed by the Pearson correlation coefficient analysis. $\mathrm{P}<0.05$ indicates that the difference is statistically significant.

\section{Results}

mRNA expression levels of apoptosis-related factors in PBMC of the two groups. The mRNA expression levels of FAS, CASP3, CASP8 and CASP9 in the observation group were significantly higher than those in the control group $(\mathrm{P}<0.05)$ (Table III).

Protein expression levels of apoptosis-related factors in PBMC of the two groups. The protein expression levels of FAS, CASP3, CASP8 and CASP9 in the observation group
Table VI. Correlation analysis of apoptotic molecules in PBMC and HBV DNA quantitative log value.

\begin{tabular}{lcc}
\hline Item & r & P-value \\
\hline FAS & 0.508 & 0.003 \\
CASP3 & 0.531 & 0.017 \\
CASP8 & 0.506 & 0.004 \\
CASP9 & 0.475 & 0.014 \\
\hline
\end{tabular}

were significantly higher than those in the control group $(\mathrm{P}<0.05)$ (Table IV).

Comparisons of positive rate of serum $H B V D N A$ and log value of copy amount of HBVDNA between the two groups. The positive rate of HBV DNA and the log value of the copy amount of HBV DNA in the observation group were significantly higher than those in the control group $(\mathrm{P}<0.05)$ (Table $\mathrm{V})$.

Correlation analysis of FAS, CASP3, CASP8 and CASP9 protein expression levels and HBV DNA quantification. The Pearson correlation coefficient analysis shows that FAS, CASP3, CASP8 and CASP9 were positively correlated with HBV DNA $(\mathrm{P}<0.05)$ (Table VI and Fig. 1).

Protein expression levels of apoptosis-related factors in $P B M C$ of patients with positive and negative $H B V-D N A$. The mRNA expression levels of FAS, CASP3, CASP8 and CASP9 in patients with positive HBV-DNA were significantly higher than those with negative HBV-DNA $(\mathrm{P}<0.05)$ (Table VII).

\section{Discussion}

As one of the most important organs that regulates body metabolism, liver is responsible for a variety of biological transformation and storage, such as storage and metabolism 

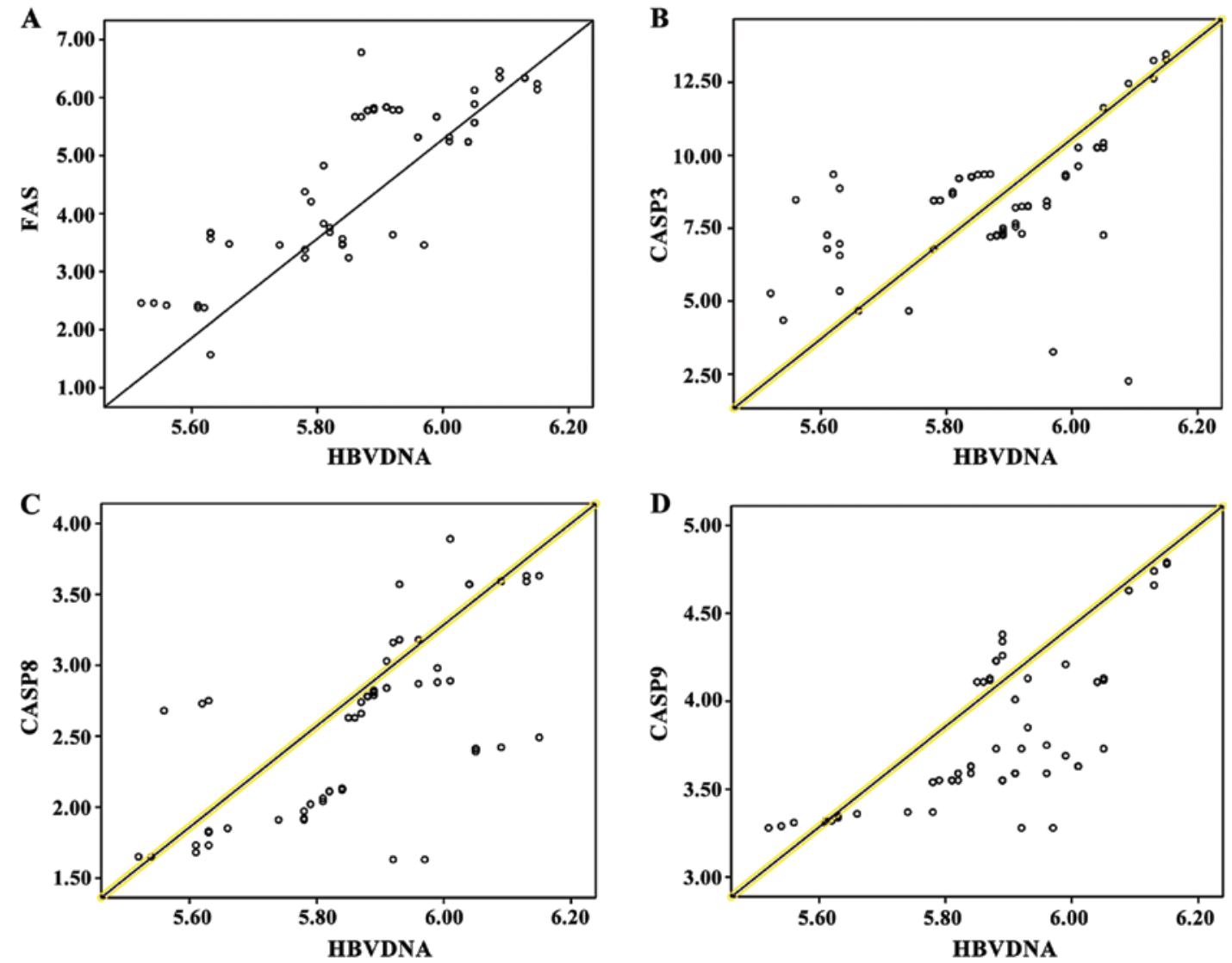

Figure 1. (A-D) The correlation analyses of FAS, CASP3, CASP8 and CASP9 protein expression levels and HBV DNA quantification, respectively; Pearson correlation coefficient analysis shows that FAS, CASP3, CASP8 and CASP9 are positively correlated with HBV DNA quantification.

Table VII. Comparisons of protein expression levels of apoptosis-related factors in PBMC between the two groups (ng/ml).

\begin{tabular}{lccccc}
\hline Group & Cases & FAS & CASP3 & CASP8 & CASP9 \\
\hline Positive HBV-DNA & 32 & $4.53 \pm 0.64$ & $7.38 \pm 0.74$ & $2.86 \pm 0.53$ & $3.35 \pm 0.53$ \\
Negative HBV-DNA & 30 & $2.34 \pm 0.35$ & $3.98 \pm 0.63$ & $1.37 \pm 0.42$ & $1.98 \pm 0.47$ \\
t-value & & 23.340 & 27.284 & 17.174 & 15.088 \\
P-value & $<0.05$ & $<0.05$ & $<0.05$ & $<0.05$ \\
\hline
\end{tabular}

of protein and fat, bile secretion, synthesis of coagulation factors and detoxification function (7). The various types of hepatitis generally occur after pathogen infection, and among them, CHB accounts for the majority of cases of hepatitis, which has the highest incidence rate of infectious diseases in China (8). CHB is usually caused by hepatitis B virus infection, and approximately $5-10 \%$ of cases transform to $\mathrm{CHB}$, and 10-20\% of CHB will progress to cirrhosis, thereby increasing the risk of primary liver cancer, eventually leading to the death of patients $(9,10)$. As one of the most basic life phenomena, cell apoptosis is an active process with steps that are regulated by the combined action of various apoptosis-related factors. Normal cell apoptosis is an important mechanism which regulates the regeneration of tissue and cells as well as the growth and development of organisms in order to maintain the stability of the internal environment. Viral infection, heart failure, neurodegenerative diseases, myocardial ischemia and other diseases can be caused by excessive apoptosis, and insufficient apoptosis will lead to tumors, atherosclerosis, autoimmune diseases and so forth. Therefore, cell apoptosis has become a hot topic in clinical research (11). Severe liver dysfunction and a large number of apoptosis in hepatocytes are the pathological features of CHB, which are manifested by the abnormal biochemical indicators in liver function.

In the process of HBV infection, lymphatic monocytes will migrate and infiltrate to the infected liver tissues, which is infected by the virus, thus leading to proliferation and activation. Apoptosis will be induced and removed by the apoptotic mechanism after being activated (12,13). PBMC-mediated antiviral immune response is an important mechanism for HBV clearance from the body, however, the continuous replication of virus and increasing viral load in CHB patients are mainly caused by the reduction of antiviral immune response function (14).

Caspase family members can play catalytic roles, which only show relatively low activity levels in the normal condition 
without any damage to normal cells and tissue, and protein substrates can be decomposed when they are largely activated, thus inducing cell apoptosis (15). FAS is a member of the tumor necrosis factor receptor superfamily, which is widely expressed in PBMCs, and initiates a caspase cascade activation process after binding to FAS ligand (FASL) (16). Apoptosis is usually carried out by the exogenous and endogenous pathway. The exogenous pathway is triggered by the binding of FAS and FASL, which leads to the activation of CASP8, and subsequently, CASP9 and CASP3 are activated. The endogenous pathway is induced by the viral protein response, DNA damage and oxidative stress, which results in the activation of CASP9 $(17,18)$. CASP3 is a member of the CASP family, which plays an important role in regulating activated T-cell apoptosis and maintaining B-cell homeostasis (19). The results of this study indicate that the mRNA and protein expression levels of FAS, CASP3, CASP8 and CASP9 in the observation group were significantly higher than those in the control group $(\mathrm{P}<0.05)$, which suggests that PBMC apoptosis can be induced by HBV infection, and FAS, CASP8 and CASP9 are activated by an exogenous and endogenous pathway and is highly expressed, resulting in the activation of the common effector, CASP3, which will combine these two pathways in order to execute the process of cell apoptosis.

The replication of HBV in the body can be reflected by the HBV-DNA content level in blood, and evidence-based medicine has shown that the hepatitis activity can be reduced by the inhibition of HBV replication, which ameliorates the prognosis of patients (20). The results of this study demonstrated that the positive rate of HBV DNA and the log value of copy amount of HBV DNA in the observation group were significantly higher than those in the control group, and FAS, CASP3, CASP8 and CASP9 were positively correlated with HBV DNA $(\mathrm{P}<0.05)$. The mechanism at play is that the body's antiviral immune response can be inhibited and reduced by apoptosis-related factors in PBMCs through the exogenous and endogenous pathway, leading to a continuous infection and replication of $\mathrm{HBV}$, which will increase the copy amount of HBV DNA and activate inflammatory reaction, thereby aggravating liver tissue injury through various inflammatory factors, such as TNF- $\alpha$, IL-6 and IL- $8 \mathrm{~h}$, which will participate in the development process of $\mathrm{CHB}$. The excessive activation of the PBMC apoptosis is closely associated with the content of HBV-DNA. The reduction of lymphocyte activity and number that is caused by excessive PBMC apoptosis will lead to an imbalance of immune function, thereby weakening antiHBV immune response. Therefore, $\mathrm{CHB}$ will be aggravated due to an ineffective clearance of HBV. This study also reveals that the mRNA expression levels of FAS, CASP3, CASP8 and CASP9 in patients with negative HBV-DNA were significantly lower than those with positive HBV-DNA $(\mathrm{P}<0.05)$, suggesting that PBMC apoptosis-related factors are inhibited via treatment and other interventions. Therefore, the illness of patients with CHB can be controlled and HBV-DNA is transformed to negative, which is conducive to prognosis.

In conclusion, apoptosis-related factors in PBMCs play important roles in the occurrence and development of $\mathrm{CHB}$, which affect the body's immune response and prognosis of patients. Therefore, intervention for their expressions can be considered to achieve the goal of complete clearance of HBV.

\section{References}

1. Zeisel MB, Lucifora J, Mason WS, Sureau C, Beck J, Levrero M, Kann M, Knolle PA, Benkirane M, Durantel D, et al: Towards an HBV cure: State-of-the-art and unresolved questions - report of the ANRS workshop on HBV cure. Gut 64: 1314-1326, 2015.

2. Papastergiou V, Lombardi R, Macdonald D and Tsochatzis EA: Global epidemiology of hepatitis B virus (HBV) infection. Curr Hepat Rep 14: 171-178, 2015.

3. Roberts H, Kruszon-Moran D, Ly KN, Hughes E, Iqbal K, Jiles RB and Holmberg SD: Prevalence of chronic hepatitis B virus (HBV) infection in U.S. households: National Health and Nutrition Examination Survey (NHANES), 1988-2012. Hepatology 63: 388-397, 2016.

4. Luo G, Feng X, Huang Y, Yi T, Wang D, Guo X, Yan H and Zhang G: Effects of antiviral therapy on the cellular immune response in patients with chronic hepatitis B. Mol Med Rep 11: 1284-1291, 2015.

5. Lamb $\mathrm{C}$ and Arbuthnot $\mathrm{P}$ : Activating the innate immune response to counter chronic hepatitis B virus infection. Expert Opin Biol Ther 16: 1517-1527, 2016.

6. Filippelli M, Garozzo MT, Capizzi A, Spina M, Manti S, Tardino L, Salpietro C and Leonardi S: Immune response to hepatitis B virus vaccine in celiac subjects at diagnosis. World J Hepatol 8: 1105-1109, 2016.

7. Himaja N and Shama SN: Herbal wealth for hepatotoxicity: A review. Asian J Pharm Clin Res 8: 3-9, 2015.

8. Gonzalez SA and Keeffe EB: Chronic hepatitis B and C: Update on therapy. Future Virol 4: 437-452, 2015.

9. World Health Organization (WHO): WHO Guidelines Approved by the Guidelines Review Committee: WHO Guidelines for the Prevention, Care and Treatment of Persons with Chronic Hepatitis B Infection. World Health Organization, Geneva, 2015.

10. The Korean Association for the Study of the Liver (KASL): KASL Clinical Practice Guidelines: Management of chronic hepatitis B. Clin Mol Hepatol 18: 109-162, 2012.

11. Lv J, Lin S, Peng P, Cai C, Deng J, Wang M, Li X, Lin R, Lin Y, Fang A, et al: Arenobufagin activates p53 to trigger esophageal squamous cell carcinoma cell apoptosis in vitro and in vivo. Onco Targets Ther 10: 1261-1267, 2017.

12. Hong CA and Nam YS: Functional nanostructures for effective delivery of small interfering RNA therapeutics. Theranostics 4: 1211-1232, 2014.

13. Zhao BB, Zheng SJ, Gong LL, Wang Y, Chen CF, Jin WJ, Zhang D, Yuan XH, Guo J, Duan ZP, et al: T lymphocytes from chronic HCV-infected patients are primed for activation-induced apoptosis and express unique pro-apoptotic gene signature. PLoS One 8: e77008, 2013.

14. Somal A, Aggarwal A and Upadhyay RC: Effect of thermal stress on expression profile of apoptosis related genes in peripheral blood mononuclear cells of transition Sahiwal cow. Iran J Vet Res 16: 137-143, 2015.

15. Mcllwain DR, Berger T and Mak TW: Caspase functions in cell death and disease. Cold Spring Harb Perspect Biol 7: a008656, 2015.

16. Karev VE: Fas, FasL, and bcl-2 expression on hepatic intralobar lymphocytes in different variants of the natural course of chronic HBV and HCV infection and in its outcomes. Arkh Patol 76: 16-21, 2014 (In Russian).

17. D'Oliveira Martins F, Gomes BC, Rodrigues AS and Rueff J: Genetic susceptibility in acute pancreatitis: Genotyping of GSTM1, GSTT1, GSTP1, CASP7, CASP8, CASP9, CASP10, LTA, TNFRSF1B, and TP53 gene variants. Pancreas 46: 71-76, 2017.

18. Meng P, Yoshida H, Tanji K, Matsumiya T, Xing F, Hayakari R, Wang L, Tsuruga K, Tanaka H, Mimura J, et al: Carnosic acid attenuates apoptosis induced by amyloid- $\beta 1-42$ or $1-43$ in SH-SY5Y human neuroblastoma cells. Neurosci Res 94: 1-9, 2015.

19. Guo X, Dong Z, Yamada S, Li Y, Guo Y, Shen S, Liang J, Tanimoto A and Guo W: Association of Casp3 microRNA target site (1049216) SNP with the risk and progress of cervical squamous cell carcinoma. Int J Gynecol Cancer 27: 206-213, 2017.

20. Peng CY, Hsieh TC, Hsieh TY, Tseng KC, Lin CL, Su TH, Tseng TC, Lin HH, Wang CC and Kao JH: HBV-DNA level at 6 months of entecavir treatment predicts $\mathrm{HBeAg}$ loss in $\mathrm{HBeAg}$ positive chronic hepatitis B patients. J Formos Med Assoc 114: 308-313, 2015. 